\title{
Gaston Contremoulins : un pionnier méconnu de la radioprotection
}

\author{
P. MORNET ${ }^{1}$
}

(Manuscrit reçu le 6 décembre 2010, accepté le 19 décembre 2010)

RÉSUMÉ Né en 1869, Gaston Contremoulins commence sa carrière comme artiste peintre avant de rejoindre à l'âge de 22 ans le laboratoire du professeur Étienne-Jules Marey, inventeur de la chronophotographie, puis les hôpitaux de Paris en 1895 au moment de la découvert des rayons $X$ par Wilhelm Röntgen. Passionné de photographie, ingénieur autodidacte ingénieux, Contremoulins publie dès 1896 avec le chirurgien Charles Remy une série de travaux sur l'usage des rayons X pour la recherche anatomique et la localisation des corps étrangers dans le crâne qui seront récompensés par le prix Montyon de l'Académie des sciences en 1897. Nommé chef des laboratoires de radiologie des Hôpitaux de Paris en 1898, Contremoulins met au point sa méthode de métroradiographie pour localisation précisément des corps étrangers dans tous les organes qui rendra d'immenses services pendant la première guerre mondiale. Très tôt conscient des dangers des rayonnements pour les médecins, le personnel hospitalier mais aussi le voisinage des postes de radiologie, Contremoulins développe des moyens de protection des sources mais aussi préconise le plombage des murs et des planchers des installations malgré l'opposition d'une partie des radiologues. Retraité des Hôpitaux de Paris, Contremoulins exercera encore pendant 16 années ses talents à l'hôpital de Saint-Germain-en-Laye. Atteint d'une cataracte inopérable, il met fin à ses jours en 1950.

ABSTRACT Gaston Contremoulins: an unknown pioneer of radiation protection.

Born in 1869, Gaston Contremoulins began his career as a painter before joining at the age of 22 years the laboratory of Professor Étienne-Jules Marey, the inventor of the time lapse, then the Paris hospitals in 1895 just when the $X$-rays were discovered by Wilhelm Roentgen. Fascinated by photography, ingenious self-taught engineer, Contremoulins published in 1896 with the surgeon Charles Remy a series of studies on the use of $\mathrm{X}$-rays for research and anatomical localization of foreign bodies in the skull. This work was awarded by the Montyon prize of the French Academy of Sciences in 1897. Appointed chief of the radiological laboratories of the Paris Hospitals in 1898, Contremoulins developed his method named "metroradiography" for locating precisely foreign bodies in all organs that made great services during the First World War. Early awareness of radiation hazards for physicians, hospital staff but also the neighbourhood of the radiological installations, Contremoulins developed ways of protecting sources, but also promoted the shielding of walls and floors of the facilities despite the opposition of some of the radiologists. Retired from the Paris Hospitals, Contremoulins exercised for another 16 years his talents in the Saint-Germain-en-Laye Hospital. Diagnosed with inoperable cataracts, he ends his days in 1950.

Keywords: History of X-rays / pioneer / radiation protection

\footnotetext{
${ }^{1}$ Ancien chef de service de médecine interne, Centre hospitalier de Saint-Germain-en-Laye, rue Armagis 78100 Saint-Germain-en-Laye, France.
} 


\section{Introduction}

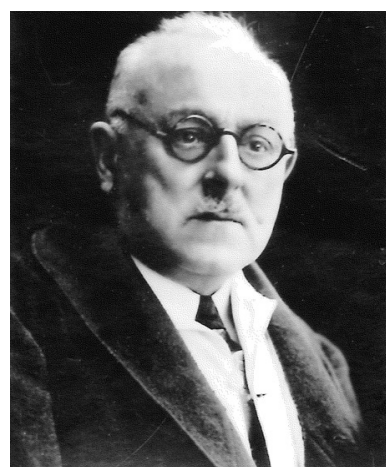

Rares sont les découvertes scientifiques à avoir suscité un tel élan créatif que la découverte des rayons «X » par Wilhelm Röntgen en novembre 1895. Quelques mois à peine après celle-ci, physiciens, médecins, photographes, industriels, commerciaux s'ingénient à en définir les applications, des plus sérieuses aux plus fantaisistes. Si l'histoire médicale a retenu en France certains grands noms des pionniers de la radiologie et de la radioprotection - Paul Oudin, Toussaint Barthélémy, Antoine Béclère, etc. - elle a, semble-t-il, omis de souligner les remarquables travaux de Gaston Contremoulins, chef du laboratoire principal de radiologie de l'Hôpital Necker de 1898 à 1934, notamment dans les domaines de la radiochirurgie et de la radioprotection.

\section{Les années d'apprentissage}

Gaston Contremoulins est né le 28 mai 1869 à Sotteville-les-Rouen dans une famille modeste. Le grand père a été mécanicien, le père serrurier, la mère couturière. À un an, il est orphelin de père, tué à la guerre et sa mère reçoit la gérance d'un modeste bureau de tabac au titre de veuve de guerre.

Malgré « de grandes facilités » scolaires, Gaston Contremoulins doit arrêter ses études au brevet élémentaire. Il passe trois ans à l'École des Beaux-Arts de Rouen, part ensuite faire son service militaire à Paris et décide de devenir «artiste peintre » à Montmartre où il s'installe. Il mène la vie de bohème d'un artiste « désargenté », s'intéresse à la photographie naissante, mais ne vend pas ses tableaux ! Sa mère l'incite à trouver un travail plus stable et c'est ainsi qu'il entre en 1890, à 22 ans, comme «préparateur » au laboratoire de microphotographie de la Faculté de médecine de Paris puis, l'année suivante, au laboratoire expérimental de physiologie du professeur Étienne-Jules Marey, membre de l'Institut.

C'est une période « fondatrice » (1891-1895) pour Gaston Contremoulins, qui fréquente ainsi un « Maître », médecin, membre de l'Académie des sciences et de l'Académie de médecine, unanimement reconnu pour un travail scientifique précurseur : la chronophotographie, ancêtre du cinéma, centrée sur l'étude des mouvements de l'homme (la marche) et des animaux (les vols d'oiseaux, la chute du chat...). Gaston Contremoulins, a "l'enthousiasme de la jeunesse », qui dira Marey a pour mission la synchronisation des divers appareils nécessaires à ces travaux qui demandent beaucoup d'attention. 
Gaston Contremoulins admire le professeur Marey, sa précision méthodologique, sa vision géométrique des images, sa sensibilité artistique, sa rigueur. Il louera son altruisme et son indifférence pour les vanités humaines. Pour un autodidacte, c'est une très bonne école de formation scientifique qui l'initie à la géométrie dans l'espace, la physique et la maîtrise de la photographie. ÉtienneJules Marey qui l'apprécie mais ne peut l'occuper à « temps plein » lui ouvre des portes : d'abord à l'Hôpital Trousseau dans le service du professeur Marc-Odilon Lannelongue puis au laboratoire d'histologie du professeur Mathias Duval à la Faculté de médecine de Paris. Imaginatif, il est ingénieux, construit lui-même ou fait construire les appareils qu'il conçoit. Le professeur Étienne-Jules Marey, lorsqu'il quitte son service en 1895, écrira : "J'ai été fréquemment en rapport avec Gaston Contremoulins dont j'ai présenté soit à l'Institut, soit à l'Académie de médecine plusieurs de ses travaux, qui sont, à mon avis, remarquables ».

\section{L'aventure des rayons $X$}

Gaston Contremoulins a 26 ans lorsque Wilhelm Röntgen découvre le 8 novembre 1895 les rayons X. Cette découverte connue en France dès le 10 janvier 1896 et qui fait «la une » des journaux comme le Petit Parisien est pour lui «une révélation ». À l'Académie des sciences, Henri Poincaré ami de Röntgen, présente le 20 janvier 1896 les toutes premières radiographies françaises de la main réalisées par Paul Oudin (1851-1923) et Toussaint Barthélémy (1850-1906) qui nécessitent à l'époque un temps de pose de 25 minutes (Poincaré, 1896), Gaston Contremoulins, dès le début 1896 crée un interrupteur à mercure permettant d'écourter le temps de pose qui est de 8 heures pour une radiographie de profil du crâne !

L'été 1896, Charles Rémy, chirurgien dans le laboratoire de Mathias Duval, et Gaston Contremoulins publient à l'Académie des sciences et à l'Académie de médecine leurs résultats de radiographies du crâne (Rémy et Contremoulins, 1896a, 1896b) puis, en novembre de la même année leurs travaux sur l'usage des rayons $\mathrm{X}$ pour les recherches anatomiques, angiologie, développement, ossification, évolution des dents (Rémy et Contremoulins, 1896c). L'année suivante, ils publient une série de notes pour les deux académies concernant la mise au point d'une méthode de localisation radiologique des corps étrangers intracrâniens à l'aide d'un « compas » de repérage qu'ils ont développé et qui sera breveté en 1897 (Rémy et Contremoulins, 1897a, 1897b, 1897c).

L'ensemble de ces travaux sur la radiologie est récompensé par la remise du prix Montyon de Médecine et de Chirurgie de l'Académie des sciences à Rémy et Contremoulins en 1897 (Rémy et Contremoulins, 1897d). Cette consécration scientifique arrive au bon moment pour Gaston Contremoulins qui n'a que 28 ans. 
En effet en 1898 les installations radioscopiques surtout, mais aussi radiographiques, se répandent en France de façon anarchique en ville et dans les hôpitaux. Chaque service hospitalier veut posséder son «installation ». À Paris, le professeur Félix Guyon, urologue réputé de l'Hôpital Necker demande à Gaston Contremoulins de lui monter « une unité radiologique » pour préciser l'existence et le siège des calculs urinaires. Cette unité lui donnera entière satisfaction.

Le conseil municipal de Paris, tuteur de l'AP-HP, s'inquiète du coût potentiel de ces mini-unités radiologiques dispersées, bien que nombre d'entre-elles soient financées par les médecins eux-mêmes. Il est alors demandé à Gaston Contremoulins de concevoir un modèle de laboratoire de radiologie plus ambitieux qui pourrait être attribué à certains grands hôpitaux de l'Assistance publique (Contremoulins, 1898, 1899).

Les rayons $\mathrm{X}$, dont on ignore encore la nature, sont à l'origine beaucoup plus accessibles aux esprits scientifiques qu'aux esprits médicaux, que leurs études sont, malgré la création d'une propédeutique scientifique en 1894, préférentiellement orientées vers la sémiologie clinique, la bactériologie (Pasteur), l'anamato-pathologie, la physiologie expérimentale (Claude Bernard). Seuls quelques grands esprits médicaux pressentent l'intérêt diagnostique et thérapeutique de tels rayons et se lancent dans l'aventure, souvent critiqués, voire moqués par leurs « collègues professeurs » de la faculté. C'est tout le mérite du professeur Antoine Béclère (1856-1939) qui écrira « cette voie m'apparut comme le chemin de la terre promise, je m'y engageai!».

L'Assistance publique et le conseil municipal de Paris approuvent le modèle de laboratoire de radiologie conçu par Gaston Contremoulins qui a l'appui de tout le corps médical de l'Hôpital Necker et créent dans un premier temps 4 laboratoires : Hôpital Necker (Gaston Contremoulins), Hôpital Salpêtrière (Albert Londe puis Charles Infroit), Hôpital Baudelocque (Charles Vaillant) tous dirigés par des «non-médecins » et un laboratoire de l'Hôpital Saint-Antoine, dirigé par le docteur Adolphe Leray, à la demande du professeur Antoine Béclère.

Ces nominations de non-médecins à la tête de ces laboratoires de radiologie des hôpitaux de Paris vont faire l'objet d'une longue polémique, initiée en octobre 1899 par Antoine Béclère (1899) et reprise par le docteur Alexandre Hennecart, un de ses élèves en 1905 au congrès radiologique de Berlin (Hennecart, 1905) où les non-médecins seront accusés "d'exercice illégal de la médecine » sans que distinction soit faite entre les noms sus-cités et les nombreux charlatans qui, en ville et dans les grands magasins, dans les foires appliquent « l'effet miraculeux des rayons $X »$, sans en mesurer les dangers. Cette polémique restera française car sans équivalent à l'étranger (États-Unis, Grande Bretagne, Suisse, ...) où 
physiciens et médecins coopèreront de façon pragmatique à la diffusion des techniques radiologiques et aux campagnes de radioprotection. Wilhelm Conrad Röntgen, le découvreur des rayons X n'était-il pas professeur de physique et non docteur en médecine !

Fort heureusement, l'Académie des sciences de 1907 à 1909 et de nombreux professeurs de médecine et de chirurgie (Jean Sicard, Pierre Delbet, Joseph Babinski...), non radiologues, en appelèrent au bon sens des responsables politiques, comme le Premier ministre de l'époque et Ministre de l'intérieur Georges Clémenceau, médecin lui-même, qui décida en juin 1909 de maintenir en place les quelques «non-médecins », physiciens de grand talent comme Gaston Contremoulins et son adjoint Puthomme, réservant aux médecins l'exercice exclusif de la radiothérapie (Clémenceau, 1907).

Parallèlement est mis en route à la Faculté de médecine de Paris, une formation théorique et pratique sous la haute autorité du professeur Antoine Béclère. L'Académie des sciences, toujours en 1909, après la visite du service de Gaston Contremoulins par 37 de ses membres, demande avec insistance aux autorités de rattacher le service de Gaston Contremoulins à l'École pratique des hautes études, lui permettant ainsi de former des élèves (Académie des sciences, 1911). Le conseil de surveillance de l'Assistance publique s'opposera à ce rattachement (Silhol, 1920).

La querelle entre médecins et non-médecins, sera relancée en 1929 par le syndicat des médecins radiologistes qui fera voter un texte à la Chambre de députés conférant aux médecins l'exclusivité de l'exercice radiologique. Une fois encore ce furent l'Académie des sciences et de nombreux médecins et chirurgiens qui soutinrent Gaston Contremoulins, dernier en piste (les autres étant décédés) et obtinrent qu'il puisse exercer son « art » jusqu'à l'âge de sa retraite de l'AP-HP en 1934.

\section{La métroradiographie et ses applications chirurgicales}

Nommé à 29 ans, chef de laboratoire auquel seront rattachés en 1898, 4 puis 13 hôpitaux de l'Assistance publique, Gaston Contremoulins doit tout créer dans des locaux vétustes. Mais rien ne le rebute et soutenu par le conseil municipal de Paris et son rapporteur Henri Rousselle, il reçoit une subvention significative pour mettre en forme et équiper le premier vrai service central de radiologie des hôpitaux de Paris. Pour atteindre cet objectif, il obtient l'autorisation d'installer un atelier de mécanique de précision, unique en son genre avec fraiseuses, perceuses, rectifieuses,... Il travaille au $1 / 100^{\mathrm{e}}$ de millimètre ! Il construit des appareils et des appareillages que les industriels de l'époque ne fournissent pas ou à des prix exorbitants. 
Dès 1903, un appareillage de radiologie et de mesure de rayons X, complexe pour l'époque, est créée et Contremoulins définit sa méthode : métroradiogaphique (Contremoulins, 1905), car soucieux avant tout de mensurations précises des pièces anatomiques et de leur situation dans l'espace. Il ne saurait se contenter de clichés de face et de profil car comme déjà souligné, dès 1896, il s'était lancé dans le très difficile travail de recherche et de localisation précise des corps étrangers intracrâniens, ensuite intra-thoraciques ou abdominaux. Il dessine des « épures », crée un compas qu'il améliorera au fil du temps. Il s'inspire de la méthode de relevé de plan de Aimé Laussedat, ingénieur militaire, topographe, géomètre (Laussedat, 1899), futur directeur du Conservatoire des arts et métiers à Paris. Ce compas rendra de grands services pendant la guerre de 14-18 (Kirmisson, 1917). En effet, en 1917, la recherche de corps étranger dure 30 à 35 minutes et le réglage du compas ne demande que 3 à 4 minutes. Ainsi, 37780 examens seront pratiqués avec l'appareillage de Contremoulins et la dernière année de la guerre, Contremoulins prendra 28 heures de garde à Necker toutes les 48 heures !

Après la guerre de très nombreux blessés souvent opérés dans de mauvaises conditions initiales, présenteront des handicaps fonctionnels considérables et douloureux par défaut de consolidation, infection, pertes de substance. Maurice Robineau, chirurgien à Necker, et Gaston Contremoulins, dont la confiance, le dévouement, la rigueur sont partagés, constituent une équipe radio chirurgicale exemplaire (Robineau et Contremoulins, 1923, 1929). Ne comptant pas leur temps, ils mettent chacun leurs talents au service des blessés de guerre abandonnés à leur triste sort. Robineau, «opérateur époustouflant » dira Clovis Vincent, a compris que les résultats postopératoires dépendent de la précision de la conception physique, géométrique et mécanique de la pièce opératoire qui doit être posée. La chirurgie orthopédique de l'après-guerre pour Robineau, ne peut plus être seulement conservatrice : elle doit être « réparatrice et reconstructive ». Gaston Contremoulins a les compétences pour cela et avec son remarquable mécanicien de Necker, Jacquart, il construit un matériel orthopédique sûr et bien toléré. Exemple remarquable : une prothèse de coude posée en $1925 \mathrm{chez}$ un homme de 20 ans par Robineau pour une tumeur bénigne inextirpable, sera retirée 60 ans plus tard par le docteur Cohen à l'Hôpital de Saint-Germain-en-Laye !

Laissons Robineau conclure la fin de ce bref exposé sur les nombreuses réalisations de Contremoulins dans le domaine de la radio chirurgie par sa déclaration à la Société nationale de chirurgie en 1927 : "Je suis certain qu'aux États-Unis, Contremoulins serait depuis longtemps à la tête d'un centre de chirurgie osseuse avec des élèves radiologues, mécaniciens, avec des collaborateurs chirurgiens. Chez nous on attendra sans doute sa mort pour reconnaître la valeur de ses travaux et les mettre en applications! ». Nul n'est prophète en son pays! 


\section{La radioprotection}

Dès mars 1896, Gaston Contremoulins constate au détour de 2 examens radiologiques prolongés ( 2 fois 50 minutes) pour localiser la balle endocrânienne d'un jeune malade de Rémy que ce dernier présente une « dermite » importante du cuir chevelu qui sera longue à cicatriser. Il attribue celle ci à l'effet délétère probable des rayons $\mathrm{X}$. Ce n'est que plus tard que Benoit prouvera scientifiquement que les réactions biologiques résultent de l'absorption par les téguments des rayons $\mathrm{X}$ les moins pénétrants (rayons mous).

En France, Marc-Odilon Lannelongue dès avril 1897 rapporte à l'Académie des sciences deux cas de lésions cutanées étendues évoluant vers la gangrène. Le 26 juin 1897 c'est le docteur Bardet qui rapporte à l'Académie les effets des rayons $\mathrm{X}$ sur la rétine (Bardet, 1897). Quant à Paul Oudin et Toussaint Barthélémy, ils présentent en août 1897 au congrès de Moscou un recueil d'une cinquantaine de cas signalés dans la littérature d'atteintes cutanées sévères (Oudin et Bartélémy, 1898). Ils notent leur survenue tardive, décalée de 15 jours à 3 semaines après l'exposition et leur lenteur de cicatrisation. Ils signalent la possibilité de formes aigües. Ils en soulignent le caractère "régressif » possible après l'arrêt de l'exposition à une source radiogène.

À l'étranger, Allen Potter en juillet 1897 pratique la première excision-greffe d'une lésion cutanée radioinduite et Elihu Thomson en 1898 démontre l'intérêt de l'utilisation d'une lame de plomb pour la protection de la peau (Thomson, 1898). La littérature y est pourtant plus alarmiste. Un dentiste de Boston, William Rollins est le premier praticien à "tirer la sonnette d'alarme »! Diplômé de Harvard, ayant de bonnes connaissances physiques, il recommande de protéger la source radiogène. Le 24 février 1901, il publie un article retentissant «X Light Kills » dans le Boston Medical et Surgical Journal (Rollins, 1901). De 1900 à 1904, il publiera plus de 200 rapports où il encourage les médecins américains à la prudence et il met en garde les femmes enceintes contre les rayons $\mathrm{X}$.

En 1902, au congrès de Vienne, Guido Holznecht présente le premier instrument de mesure quantitative des rayons de Röntgen. Après lui, Heinrich Albers-Schönberg de Hambourg expérimente l'effet des rayons X sur les animaux (lapins, cobayes) et obtient une stérilité par mort des spermatozoïdes. Le docteur Henricke de Leipzig après une irradiation massive de souris et de jeunes cobayes constate de très importantes lésions histologiques entraînant la mort en quelques heures des lymphocytes folliculaires de la rate et de tous les groupes ganglionnaires. Une atteinte de la moelle osseuse est observée plus tardivement (Albers-Schönberg, 1904). 
À Paris, Antoine Béclère rappelle la nécessité d'apprécier la qualité et la quantité des rayons de Röntgen émis et il publie dans la revue le radium un long article sur « les moyens de protection du médecin et des malades contre l'action nocive des nouvelles radiations : rayons de Röntgen et rayons de radium ». Il décrit la cabine mobile de plomb d'Albers-Schönberg tel un « commandant de navire de guerre » dans sa tourelle blindée, certes très efficace mais jugée incommode et il semble lui préférer les moyens de protection tels que les gants et tabliers de plomb, lunette en verre plombé, l'enfermement de la source radiogène dans une coquille de cuivre ou de plomb et une coquille de cuivre pour tenir le manche du chalumeau (Béclère, 1904).

Gaston Contremoulins qui a lu toutes ces publications considère les rayons $\mathrm{X}$ qui traversent le tube de Crookes et impriment une plaque photographique « à distance » comme des rayons potentiellement dangereux pour toute personne se trouvant à une distance proche de la source radiogène ». Il avait très tôt identifié les risques pris par les opérateurs qui exposaient leurs mains pour apprécier l'intensité des rayonnements émis. Il va baser sa stratégie de radioprotection en s'appuyant sur 2 axes :

$\mathbf{1}^{\text {er }}$ axe : un contrôle des sources de rayons X par un appareillage rigoureux et précis pour protéger le radiologue et le personnel, d'autant mieux qu'il maîtrise parfaitement ce matériel qu'il a en grande partie construit lui-même dans son atelier de mécanique de précision. Il privilégie cette démarche à l'utilisation d'habits plombés, dignes d'un scaphandrier, chers et incommodes et impressionnants pour les patients. Certes les radiologues sont protégés, mais leur personnel...?

$2^{\mathbf{e}}$ axe : un isolement rigoureux des lieux d'émissions des rayons X par plombage des murs et des planchers afin de protéger le voisinage.

Le premier axe est donc la maîtrise technique et rationnelle de l'installation radiologique. Contremoulins a, comme on l'a vu, équipé son laboratoire d'un appareillage métroradiographique qu'il perfectionne au fil du temps, considérant qu'il y a " antinomie entre les soucis de rentabilité des constructeurs et celui de spécialistes ». Il ajoute avec humour : "L'autruche croit se protéger du chasseur en s'abritant la tête sous une pierre. L'enfant se cache derrière une porte pour éviter la balle ou le fusil. Le radiologiste n'est guère mieux protégé par les moyens que l'industrie lui fournit. »

Il lui importe donc de mesurer la qualité de pénétration et la quantité de rayons $\mathrm{X}$ émis en un temps de pose défini et contrôlé. Il préfère améliorer les performances du radiochronomètre de Benoist avec son métro-radioscope en 1902 qu'il fait breveter, compléter par le radiophotomètre et bien entendu l'indispensable 
osmorégulateur de Paul Villard qu'il adapte pour le contrôle du vide des tubes émetteurs. Enfin, il règle le temps de pose avec la construction d'un Tachydécompteur disjoncteur automatique. Il se repose sur sa table de métro-radiographie où la source radiogène bien protégée dans sa coquille plombée, émet des rayons précisément focalisés sur la zone ciblée et où le patient est immobilisé par des gouttières plombées qui évitent ainsi la diffusion latérale des rayons secondaires. Enfin, il met au point avec la direction de Saint-Gobain, une cloison isolante fixe pour l'opérateur. Celle ci comporte un soubassement en bois de $2 \mathrm{~m}$ de largeur sur $1 \mathrm{~m}$ de hauteur, au-dessus duquel est fixée une dalle de cristal plombée de même grandeur $(2 \mathrm{~m} \times 1 \mathrm{~m})$ d'une épaisseur de $25 \mathrm{~mm}$ permettant de surveiller le patient et les divers appareillages de mesure.

Ce premier axe de la politique de radioprotection, définie par Contremoulins est rapidement et facilement réalisé. Il maîtrise sa « technologie radiogène ». Le deuxième axe est beaucoup plus difficile à atteindre rapidement car il dépend des investissements financiers que voudra bien lui allouer l'Assistance publique où déjà il se heurte à une opposition «sourde » mais réelle des autres radiologistes, qui à partir de 1910 seront tous médecins.

Dès 1911, Contremoulins avait demandé une protection par plombage des murs et du sol de sa salle principale de radiologie avec des plaques de plomb de 3 mm d'épaisseur. Avec l'apparition du tube de Coolidge en 1913 beaucoup plus puissant que les tubes de Crookes Contremoulins insiste sur le «renforcement» nécessaire des mesures d'isolement et il préconise désormais des plaques de $5 \mathrm{~mm}$ d'épaisseur pour plomber les murs et les sols. Il a dû lui-même garnir avec une plaque de $10 \mathrm{~mm}$ l'un des côtés de sa cabine isolante. D'après Contremoulins de tels protecteurs "sont 4000 fois plus efficaces que les capsules les mieux protégées et doivent être généralisés à tous les services radiologiques de l'assistance publique » (Contremoulins, 1911).

À partir de 1920, il alerte régulièrement l'Assistance publique dans ses rapports annuels sur les dangers d'un retard à l'exécution des travaux de plombage, même si à cette époque après 132000 examens pratiqués, personne dans son service n'est atteint physiquement (Contremoulins, 1920-1929). Toutefois l'hécatombe des premiers utilisateurs de rayons $\mathrm{X}$ et de leur personnel est déjà alarmante : Blanche Weitman, infirmière à la Salpêtrière, Charles Infroit qui décède en 1920, Charles Vaillant amputé des doigts, avant bras, bras, Adolphe Leray de l'hôpital SaintAntoine qui a dû « céder un pouce au scalpel » et qui décèdera en 1921. Ces victimes des rayonnements s'ajoutent à celles de l'étranger: Clarence Dally (1904), Louis Weigel (1906), Heinrich Albers-Schönberg (1921) et bien d'autres encore ! Après la mort d'Adolphe Leray, Contremoulins se révolte, outré par ces cérémonies à l'hôtel de ville de Paris où l'on remet des décorations à des praticiens 
à qui l'on n'a pas donné - ou ordonné - de mettre en place les moyens d'une protection efficace.

Mais le pire est à venir avec l'installation désordonnée de cabinets de radiologie et de radiothérapie dans des habitations urbaines. Il considère qu'il existe un véritable danger sociétal. Il s'en suit une série de lettres adressées par Contremoulins au directeur de l'AP-HP pour accélérer le plombage des installations radiologiques. Le directeur répond en " nommant» une commission dont le rapporteur le docteur Joseph Belot ne voit « a priori rien qui justifie une telle demande » et conseille qu'une étude sérieuse soit faite pour la justifier. Gaston Contremoulins qui n'a aucune confiance en cette commission décide de pratiquer au plus vite « cette étude sérieuse » et de la soumettre à la non moins sérieuse «Académie des sciences » où il se sait écouté et estimé.

Il utilise le tube de Coolidge avec des intensités moyennes de $17 \mathrm{~cm}$ de longueur d'étincelle, 2 milliampères, temps de pose 60 minutes, et il imprime à 4,60 m des objets opaques (crâne, omoplate) situées dans la salle située à l'étage en dessous de la source radiogène, à travers une plaque de marbre de $28 \mathrm{~mm}$, une feuille de plomb de $3 \mathrm{~mm}$, un plancher de $30 \mathrm{~cm}$, constitué de chêne et $10 \mathrm{~cm}$ de hourdis de plâtre... Il utilise une intensité de rayons correspondant à un examen radioscopique ou radiographique standard, mais n'ose imaginer ce que donnerait une pareille étude avec les sources radiogènes utilisées en radiothérapie profonde (25 cm d'étincelle, 12 à 15 heures de temps de pose). Il affirme que « de telles sources d'énergie ne peuvent être installées au centre des agglomérations urbaines, sans faire courir les plus graves dangers aux habitants et des mesures de précaution s'imposent de toute urgence ». Il conclue à la nécessité d'utiliser des plaques de plomb de $6 \mathrm{~mm}$ situées à $2 \mathrm{~m}$ du foyer radiogène (Contremoulins, 1921a).

Sa communication à l'Académie des sciences soulève une émotion compréhensible et Contremoulins pour enfoncer le clou, alerte le Ministère de l'hygiène, de l'assistance et de la prévoyance sociale. Il demande avec insistance que soit confiée à l'Académie la mission de mesurer précisément avec les techniques adéquates (ionisation, ...) la nature et la quantité de rayonnement qui traverse les murs et les planchers. Contremoulins poursuit sa démonstration en augmentant la distance des plaques de métro-radiographie de la source radiogène (40 m et $80 \mathrm{~m})$ à travers les murs d'un bâtiment situé en face de son service à Necker. Il utilise le même courant : $17 \mathrm{~cm}$ d'étincelle, 2 milliampères, avec un temps de pose de $1 \mathrm{~h}$ et de $4 \mathrm{~h}$ (Contremoulins, 1921b).

Les images obtenues d'os, d'une bouteille de verre, d'un crabe mais aussi de ficelles de chanvre, de compresses de lin à poids atomique faible sont identiques aux images des expériences précédentes. Il considère que le faisceau de rayons $\mathrm{X}$ 
a conservé son hétérogénéité et que donc on ne peut nier «a priori » leur action biologique à distance. Le Ministre de l'hygiène ne confie pas la mission à l'Académie des sciences mais à l'Académie de médecine probablement sous la pression d'un lobby radiologique, mieux représenté.

L'Académie de médecine qui n'a pas eu connaissance d'accident survenu à des tiers, préconise de baisser les doses au-dessous du seuil d'action nocive, sans cependant définir ce seuil. Le rapporteur conclue, persiflant « si quelques uns souffrent de leur dévouement, les vieux radiologistes sans doute, malgré les précautions connues, ils sont rares et nous les accompagnerons de notre respect et de notre reconnaissance ». Réponse d'un syndicat d'intérêt professionnel tonne Contremoulins : "J'ai tout lieu de penser qu'il y a mieux à faire! ».

Contremoulins écrit et obtient enfin du directeur de l'Assistance publique l'accord pour le plombage de son service qui sera par la suite étendu aux autres services, malgré l'avis « inique » de l'Académie de médecine. D'ailleurs, au fil des mois, inquiets sans l'avouer, les radiologistes médecins accentueront leur protection, certains suggèreront même de substituer au plombage un système d'isolement des bâtiments par des briques remplies de baryte (Béclère et al., 1922).

En 1923, à l'occasion d'une cérémonie de décoration en l'honneur de Charles Vaillant, chef du service radiologique de Lariboisière, mais aussi victime des rayons X et à ce titre promu au titre de «Martyr de la Science ! », Contremoulins relance la controverse en écrivant au président du conseil municipal de Paris, que les radiologistes sont un danger permanent, quoique méconnu, pour les habitants de Paris. Une loi s'impose ! Une nouvelle commission est diligentée par le Ministère de l'hygiène et confiée à des radiologues qui ne nient plus que les rayons de Röntgen traversent murs et plafonds mais considèrent que ces radiations sont «sans conséquence» sur les organismes humains. Il s'agit toujours d'une affirmation « a priori » sans preuve scientifique car l'Académie des sciences n'a pas effectué les études demandées. Pourquoi ? se demande Contremoulins qui se rend bien compte qu'il existe une antinomie évidente à demander à un homme ou à un groupement d'hommes de se condamner lui-même « mais c'est une question de droit qui se pose !». Dans de telles conditions, un particulier radiologue ou radiothérapeute, s'arroge le droit de soumettre autrui à l'action de radiations nocives, même à faibles doses. La commission en concluant par « a priori » ne dépasse-t-elle pas son droit strict ? N'y a-t-il pas un étrange abus de droit ? N'y at'il pas violation des obligations d'interdépendances qui régissent les rapports entre citoyens ? Si le radiothérapeute se protège mieux, comme le souligne la commission, qu'en est-il pour le personnel ? Pour les voisins ? Contremoulins milite au nom de la prudence, ce que nous appelons aujourd'hui le principe de précaution. Les commissions sont souvent créées pour enterrer les problèmes, le 
silence retombe mais lorsque les convictions sont profondes, les problèmes réapparaissent. C'est ainsi qu'en 1929, la question de la protection des tiers contre les rayons $\mathrm{X}$ est relancée. Une nouvelle commission où siège pour la première fois Contremoulins est mise en place. Il rédige un nouveau rapport, mais une fois encore, il n'est rien décidé et les positions de 1921 et de 1923 sont confirmées.

Il ne reste plus à Gaston Contremoulins qu'à avertir le grand public des dangers qui le menacent, d'où une grande campagne de presse dans Le Matin (14, 18, 19 , 20, 23 et 26 septembre 1929), Le Quotidien (21 et 24 septembre 1929), Le Siècle Médical $\left(1^{\mathrm{er}}\right.$ octobre, $1^{\mathrm{er}}$ et 15 novembre $1929,1^{\mathrm{er}}$ janvier 1931), L'Intransigeant (4 octobre 1929 et $1^{\text {er }}$ janvier 1930), Le Coopérateur de France (12 octobre 1929), Le Figaro (21 octobre 1929)... où s'opposent ouvertement : les alarmistes (Contremoulins et le conseil municipal de Paris) les dubitatifs prudents (Pr de Broglie) et les optimistes de façade (les radiologues) qui continuent d'affirmer que le danger des rayons $\mathrm{X}$ pour les voisins est un mythe !

Pourtant les travaux sur la question ne manquent pas. Il y a d'abord l'article du docteur Lassueur de Lausanne dans la Revue Médicale de Suisse Romande qui vient confirmer l'opinion défendue par Contremoulins (Lassueur, 1927). Ce médecin dermatologue et radiothérapeute à l'occasion des indications de l'époque, souligne à la lumière de son observation personnelle qu'il détaille les dangers des petites doses lentement répétées sur le système hématopoiétique et le système nerveux sympathique. Il fustige l'inconscience coupable de certains radiothérapeutes qui exposent leur personnel et leurs voisins en ne les protégeant pas contre les rayonnements secondaires. Il réclame une loi coercitive en Suisse et s'étonne que la France n'ait pas de réglementation législative, à l'image d'autres pays comme les États-Unis (AJER, 1922) ou l'Angleterre, qui a mis en place en 1921 un X-Ray and Radium Protection Committee, et qui ont établi des règles strictes de contrôle de plombage des installations radiologiques et surveillance des radiologues, de leur personnel et de leurs voisins par un contrôle de la numération formule sanguine tous les trois mois.

Par la suite, l'Allemagne, l'Autriche, la Russie, la Hollande, les pays Nordiques s'inspireront des directives anglaises Il y a aussi les travaux du docteur Jaulin présentés en 1927 au congrès pour l'avancement des sciences qui soulignent le danger des rayonnements secondaires notamment au cours de la radiothérapie profonde, préconisant un plombage absolu des murs, des plafonds, des planchers car un plancher ordinaire ne saurait suffire à faire perdre aux radiations qui les traversent toute action biologique et qu'il est erroné d'invoquer la loi du carré de la distance (Jaulin, 1927). 
Au congrès de Stockholm en 1928 sera mis en place le Comité international de protection contre les rayons $\mathrm{X}$ et le radium, rebaptisé après la deuxième guerre mondiale la Commission internationale de protection radiologique (CIPR), qui s'intéressera dans un premier temps à la radioprotection des travailleurs exposés et définira une « dose maximale admissible annuelle (DMA)» fixée au 1/10 de la dose érythème c'est-à-dire à environ 500 mSv/an.

Le docteur Lassueur de Lausanne verra ses troubles névritiques majorés gravement, le jour où s'installera sous son propre cabinet, un poste de radiothérapie non protégé par un plombage. Mon observation écrira-t-il a valeur d'expérience (sérieuse) et de citer le professeur Jean Bergonié de Bordeaux, autre victime des rayons $\mathrm{X}$, qui a décrit l'anaphylaxie physico-chimique de ces derniers.

L'intervention du docteur Lassueur entraînera un texte législatif en Suisse. En France, Léon Bouchacourt et Henry Morel-Kahn, en février 1928, réclameront « la création d'un organisme indépendant composé de médecins radiologistes, de physiciens pour contrôler les appareils de protection dans les hôpitaux et les cabinets privés » à la Société de radiologie médicale de France.

\section{L'âge de la retraite}

En 1934, Gaston Contremoulins a atteint l'âge de la retraite mais il se trouve encore de nombreux médecins et chirurgiens qui ne veulent pas le laisser partir et signent une pétition solennelle pour son maintien à l'Hôpital Necker. Après 270000 clichés, il n'a souffert que d'une discrète lésion unguéale, contractée pendant la guerre avec le matériel militaire. Aucun membre de son service n'a souffert des rayons $\mathrm{X}$ grâce à l'efficacité de sa politique de radioprotection. Pot de terre contre pot de fer ! L'administration rejette cette demande et fort heureusement pour les Saint-Germanois, Gaston Contremoulins quitte l'AP-HP pour venir diriger à l'hôpital de la ville un service de métro-radiographie, crée pour lui grâce à l'initiative et à la générosité de deux chirurgiens, les docteurs Jean-Paul Lamare, orthopédiste et le docteur Paul Larget, chirurgien viscéral qui connaissent l'aide inestimable que sa présence va apporter à leur hôpital.

De 1934 à 1950, aidé par sa collaboratrice de toujours Madame Becker et de son fidèle mécanicien Jacquart, Contremoulins réinstalle tout l'appareillage métro-radiographique qui lui appartient. Il développe l'activité urologique, la radiochirurgie et installe l'électrocardiographie absente jusqu'alors de l'hôpital. Il étudie et dépose un brevet pour la construction d'un plan de couchage original, permettant d'assurer aux opérés un meilleur confort, une meilleure hygiène et une facilité pour les soins infirmiers. Tout ce travail à temps plein lui laisse encore le temps d'écrire et d'illustrer un ouvrage important de 2 volumes, où sont exposés 
ses différents travaux de recherche appliquée. Terminé en 1943, en pleine guerre, il ne pourra être publié par les éditions Martial-Haeffelin de Paris : il ne le sera jamais par la suite.

Atteint d'une cataracte inopérable, il voit «venir » la cécité ! Sa décision est prise depuis longtemps, ayant toujours affirmé qu' inutile à la Science, sa vie n' aurait plus de sens. Le 19 novembre 1950, il se dirige à pied vers son service de l'hôpital, écrit sur son bureau son dernier message : "J'ai consacré toute ma vie à soulager la société, mais mon infirmité ne me permettant plus de continuer mes travaux et d'être utile à l'humanité, je préfère me donner volontairement la mort! ». Il a programmé ses obsèques. Il sera conduit au cimetière de Saint-Germain dans le «corbillard des pauvres » sans fleurs ni couronnes.

\section{En guise de conclusion}

La vie de Gaston Contremoulins est passionnante et son œuvre exemplaire. Sur le plan humain et professionnel, issu d'un milieu modeste, sans diplôme scientifique, il a su s'imposer tel un homme de la Renaissance, par l'étendue de ses talents : dessinateur, écrivain, scientifique, mécanicien, humaniste. Sa rigueur, son honnêteté, sa foi dans les bienfaits de la science, s'inscrivent tout à fait dans l'esprit des pionniers de son époque. Mais il a eu quelque chose de plus : son altruisme, son désintéressement personnel, financier et honorifique. À ceux qui l'incitaient à faire des études de médecine pour couper court à l'antinomie des médecins radiologistes, à qui il n'avait pas eu «l'heur de plaire», il répondait avec hauteur "pourquoi le ferais-je ? J'y perdrais de précieuses années où j'ai mieux à faire dans mes recherches. Je ne cherche pas la gloire, le prestige, la notoriété sociale (il refusera toutes les décorations). Je reste à ma place de chercheur scientifique où je me sens bien, utile à la médecine, à la chirurgie donc aux malades et c'est pour moi : l'essentiel. ». Sur le plan de la radioprotection, Gaston Contremoulins a indéniablement fait partie des courageux pionniers qui ont su, à leur époque, utiliser avec prudence à des fins diagnostiques et thérapeutiques des rayons de nature encore inconnue, réputés dangereux, sans connaître précisément le point d'équilibre entre l'objectif médical poursuivi et le risque encouru pour le praticien, le personnel et l'environnement.

\section{RÉFÉRENCES}

Académie des sciences (1911) Lettre des membres de l'Académie des sciences au ministre de l'Instruction publique et des Beaux Arts pour rattacher le laboratoire de radiographie de Necker à l'École des hautes études, 1911.

AJER (1922) American Journal of Electrotherapy and Radiology XI.

Albers-Schönberg H. (1904) Röntgenbilder. Eine seltene Knochenerkrankung, Münchner Medizinische Wochenschrift 51, 365-368. 


\section{GASTON CONTREMOULINS : UN PIONNIER MÉCONNU DE LA RADIOPROTECTION}

Bardet G. (1897) Séance de l'Académie des sciences du 14 juin 1897.

Béclère A. (1899) La radioscopie et la radiographie dans les hôpitaux, Presse médicale 84.

Béclère A. (1904) Les moyens de protection du médecin et des malades contre l'action nocive des nouvelles radiations : rayons de Röntgen et rayons du Radium, Le radium 1, 133-143.

Béclère H. et al. (1922) Présentation à la Société de chirurgie de Paris par M. Pierre Duval : Nouveau matériel de protection contre les rayons X, 25 janvier 1922.

Clémenceau G. (1907) Lettre du Président du Conseil, Ministre de l'Intérieur au Secrétaire perpétuel de l'Académie des sciences, 15 octobre 1907.

Contremoulins G. (1898) Rapport sur la radiographie dans les hôpitaux, 28 pages, éditions Gautherin, écrit dans le service du Pr Guyon, T.C. XXXII.

Contremoulins G. (1899) Création du Laboratoire Central de Radiographie de l'hôpital Necker, Chef de Laboratoire, rapports de l'exercice 1899.

Contremoulins G. (1905) Méthode générale de métroradiographie, Mesure des rayons-X, Annales d'électrobiologie et de radiologie 2.

Contremoulins G. (1911) Rapport d'exercice du laboratoire principal des hôpitaux de Necker.

Contremoulins G. (1920-1929) Rapports d'exercice du laboratoire principal des hôpitaux de Necker, 1920 à 1929.

Contremoulins G. (1921a) Note à l'Académie des sciences présentée par A. Lippmann : À propos de la protection des tiers contre les rayons X, 18 avril 1921.

Contremoulins G. (1921b) Note à l'Académie des sciences présentées par M. Lippmann : À propos de la protection des tiers contre les rayons X (Rayonnements à distance), 8 mai 1921.

Hennecart A. (1905) L'emploi des rayons Röntgen chez l'homme est uniquement du ressort médical, Congrès de Berlin, 30 avril-3 mai 1905.

Mr. Jaulin (1927) Journal de radiologie et d'électrologie XI, 193.

Kirmisson E. (1917) De l'emploi du compas de Contremoulins réglé d'après une localisation radioscopique, Académie de Médecine, 17 avril 1917.

Lassueur (1927) Radionévrite et cardiopathie réflexe, Revue Médicale de la Suisse Romande 9.

Laussedat A. (1899) La métroradiographie, Gauthier Villars, 52 pages.

Oudin P., Barthélémy T. (1898) France Médicale 8-12.

Poincaré H. (1896) Note à l'Académie des sciences: Photographie de la main obtenue à l'aide des « X-Strahlen » de Mr. le Professeur Röntgen, 20 janvier 1896.

Rémy C., Contremoulins G. (1896a) Note à l'Académie des sciences présentée par le Prof. Marey : Endographie crânienne au moyen des rayons de Röntgen, 27 juillet 1896.

Rémy C., Contremoulins G. (1896b) Note à l'Académie de Médecine: Présentation de deux radiographies du crâne, 18 août 1896.

Rémy C., Contremoulins G. (1896c) Note à l'Académie des sciences présentée par le Prof. Marey : Emploi des rayons-X pour les recherches anatomiques, angiologie, développement, ossification, évolution des dents, 2 novembre 1896.

Rémy C., Contremoulins G. (1897a) Note à l'academie de médecine présentée par le Prof. Marey : Sur un nouveau perfectionnement des applications chirurgicales des rayons $\mathrm{X}$, Recherche de projectiles dans le crâne, 30 mars 1897.

Rémy C., Contremoulins G. (1897b) Note à l'Académie de Médecine présentée par le Prof. Marey : Appareil destiné à déterminer d'une manière précise au moyen des rayons $\mathrm{X}$ la position des corps étrangers dans le crâne, 22 novembre 1897. 


\section{P. MORNET}

Rémy C., Contremoulins G. (1897c) Académie de Médecine : Extraction de projectiles contenus dans le crâne dont le siège a été déterminé par la méthode de Rémy et Contremoulins, 23 novembre 1897.

Rémy C., Contremoulins G. (1897d) Académie des sciences, Lauréats du prix Montyon de Médecine et Chirurgie, Présentation de 2 atlas de radiographie, 1897.

Robineau M., Contremoulins G. (1923) Ostéosynthèse des fractures récentes transcervicales du col du fémur, Presse Médicale, 24 septembre 1923.

Robineau M., Contremoulins G. (1929) Note à l'Académie des sciences présentée par M. Bazy, Exemples de synthèses et de prothèses en os, en métal nu ou caoutchoutées établies sur des donnés métroradiographiques, 10 juin 1929.

Rollins W. (1901) X light Kills, Boston Medical and Chirurgical Journal.

Schönberg A. (1903) Munich Med. Wochenns, 27 octobre 1903, semaine médicale du 4 novembre 1903.

Mr. Silhol (1920) Conseil de Surveillance de l'Assistance Publique, Rapport, 24 février 1920.

Thomson E. (1898) American X-Ray Journal. 